\title{
ACCURATE REAL-TIME DISPARITY MAP COMPUTATION BASED ON VARIABLE SUPPORT WINDOW
}

\author{
Nadia Baha, Slimane Larabi \\ Computer Science Department, University of Science and \\ Technology USTHB, Algiers, Algeria \\ \{nbahatouzene, slarabi\} \& usthb.dz
}

\begin{abstract}
We propose in this paper a real-time dense stereo matching algorithm using variable support window for disparity map computing. Basic real-time local algorithms relying on a fixed and rectangular correlation window suffer from the difficulty for window-based methods lies in determining the best window shape and size for each pixel. This work proposes a novel local approach using a combination of the DSI (Disparity Space Image) structure and gradient information. Two improvements are introduced so that an accurate and fast result will be reached. The first one concerns the proposition of a new strategy in order to optimize the computation time of the initial disparity map. The second one, concerns the pixel similarity measure for matching score computation and it consists to use in addition to the traditional pixel intensities, the magnitude and orientation of the gradients providing more accuracy. Experimental results on real data sets are conducted and a comparative evaluation of the obtained results relatively to the state-of-art methods is presented.
\end{abstract}

\section{KEYWORDS}

Support window, Disparity map, Disparity Space Image (DSI), Occlusion, Real-Time.

\section{INTRODUCTION}

Stereo matching is a problem to find correspondences between two or more input images. It is one of fundamental computer vision problems with a wide range of applications, and hence it has been extensively studied in the computer vision field for decades. Stereo matching consists to find for each point in the left image, its corresponding in the right one. The difference between horizontal distances of these points is the disparity. A disparity map consists of all the possible disparity values in an image. Such a map is basically a representation of the depth of the perceived scene. Therefore, the disparity maps have been used to address efficiently problems such as 3D reconstruction, positioning, mobile robot navigation, obstacle avoidance and many other domains.

Despite the simplification brought by the epipolar geometry, the problem of matching remains difficult to solve due to occlusion, luminosity changes between viewpoints and non textured areas. To overcome these difficulties, several methods have been proposed.

In general, stereo algorithms can be categorized into major classes: local methods and global methods. Local algorithms which are based on a correlation criterion can have very efficient implementations that are suitable for real-time application [1-7]. One of the principal factors which influence the success of local methods is the proper selection of a window shape and size. 
The windows must be large enough to capture intensity variation for reliable matching but small enough to avoid the effects of projective distortions at the same time. An appropriate window selection should improve matching accuracy but require an optimised balance between the above opposite criteria [8]. Global approaches minimize an overall cost function that involves all the pixels of the image. In these methods, calculating the disparity field is led to minimize the objective function of energy. Several optimization methods have been proposed such as dynamic programming [9], graph cuts [10], directed anisotropic diffusion [11], belief propagation [12], [13] and neural network based approaches [15], [16]. The global methods can generate high-quality disparity maps. However, these methods are often computationally expensive and involve difficult parameter adjustment procedures that require a lot of effort to find the optimal ones, making them unsuitable for most interactive applications. Also, there are many other methods that are not strictly included in any of these two broad classes, as example, we can cite [17-19]. A survey for the different approaches can be found in [20-22].

Given a pair of rectified stereo images $I_{r}, I_{t}$, the problem of stereo correspondence is to find for each pixel of the reference image $I_{r}$ the correspondent pixel in the target image $I_{t}$. By assuming that the images pairs are rectified, the correspondence for a pixel at coordinate $(x, y)$ can only be found at the same vertical coordinate $y$. The basic local approach selects, as the best correspondence for a pixel $p$ on $I_{r}$, the pixel of $I_{t}$ which yields the lowest score of a similarity measure computed on a (typically squared) fixed support (correlation window) centered on $p$ and for each of value of the disparity range.

The traditional local approach assumes that all pixels in support window have similar depth. However, this assumption does not hold in most real environments with large depth variation due to their structure and objects and the use of a fixed support is prone to errors due to the fact that it blindly aggregates pixels belonging to different disparities.

This paper proposes a novel adaptive support aggregation strategy which deploys DSI (Disparity Space Image) and gradient information in order to increase the reliability of the matches. By means of experimental results we demonstrate that this approach is able to improve the quality of the disparity maps compared to the state of the art of local stereo algorithms.

The main contributions of this work are:

- The use of an adaptive support window.

- The extension of matching primitives from pixel intensity to intensity, gradient magnitude and orientation of gradient vector of pixel.

- The proposition of a robust matching cost based on the combination of the DSI structure and gradient information aiming at high efficiency and at the same time as accurate as to improve the results of fast local stereo algorithms.

This paper is organised as follows: section 2 presents the related work in the field of real-time based stereo vision. Section 3 presents the stages followed to compute the initial disparity map. Section 4 presents the refinement method. In section 5, experimental results obtained on real images are presented and discussed. Finally, section 6 concludes the paper with some remarks.

\section{RELATED WORK}

Stereo vision is a very broad topic which has been extensively surveyed by [20-22]. The work [20] further illustrated the intuitive notion that while local techniques excel at achieving high speeds, global techniques are better suited to generate high quality disparity maps. 
Consequently, most recent work has focused on developing global algorithms. But significant work has also been done on local methods.

In this section, we present an overview of stereo algorithms reported in the literature. The method proposed by [23] is based on the use of ZNCC as matching cost, integrated within a neural network model. The results obtained are satisfactory, but they are not suitable for real time applications because the running time needed for standard image sets is very high. The method reported in [4] performs interval matching instead of pixel matching. The execution time of the algorithm varies from 1 to 5 seconds for the standard image sets. A window-based method for correspondence search is presented in [24] which use varying support-weights. The support-weights of the pixels in a given support window are adjusted based on color similarity and geometric proximity to reduce the image ambiguity. The running time for the Tsukuba image pair with $35 \times 35$ pixels support window is about one minute. In the method based on the Bayesian estimation theory described in [25], the results are encouraging in terms of accuracy but they are not suitable for real time applications, since it takes few minutes to process a $256 \times 255$ stereo pair with up to 32 disparity levels. The method developed in [26] uses graph cuts which produces semi-dense disparity map. The running times obtained for the Tsukuba pair is about 6 seconds and 13 seconds for the Sawtooth pair. An improvement of the aggregation strategy based on color image segmentation [7] has been proposed by [5]. The processing time achieving by this method is around 0.2 second for Tsukuba with a disparity range of 16 pixels. For the cost aggregation method presented in [6], the running time for the Tsukuba image pair is 13 seconds and 37 seconds for Teddy image pair. Another method reported by [9] uses a twopass dynamic programming technique combined with generalized ground control points (GGCP), which is designed to resolve the inconsistency between scanlines which is the typical problem in conventional dynamic programming. The processing time achieving by this method is around 4.4 second for Tsukuba image pair with a disparity range of 16 pixels. In another method reported in [16] based on Self-Organizing Neural Network, the average execution time is approximately 100 seconds for the standard image sets.

As reported in a recent paper [22], local approaches that are state-of-the art in terms of accuracy are based on segmentation [27] or adaptive weights [24], but are far from being computationally efficient. Indeed, apart from GPU or hardware-based implementation, typically only aggregation strategies based on sets of rectangular windows [1, 26,29] can afford real-time or near-real time processing, this implying a notably reduced accuracy of retrieved disparities. Exceptions are represented by methods [7,30], whose aggregation strategies rely on segmentation and that exhibit interesting trade-offs between accuracy and computational efficiency (see [22] for review).

The idea which motivates this work is to propose a novel aggregation cost deploying the DSI (Disparity Space Image) data structure and gradient information aiming at low computation time and at the same time as accurate as to improve the results of fast local stereo algorithms. This leads us to yield a level of accuracy comparable to that of global methods and able to meet near-real time processing requirement.

\section{INITIAL DISPARITY MAP ESTIMATION}

Basically, a stereo matching algorithm is built up as follows: First, usually pre-processing functions are applied, e.g. a noise filter. Second, the matching costs for each pixel at each disparity level in a certain range (disparity range) are calculated. The matching costs determine the probability of a correct match. The smaller the costs, the higher the probability. Afterwards, the matching costs for all disparity levels can be aggregated within a certain neighborhood window (block).

The idea of a variable support is mainly motivated by depth discontinuities: in order to detect accurately depth borders, the support should separate "good" pixels, i.e. pixels at the same 
disparity as the central pixel, from "bad" pixels, i.e. pixels at a different disparity from the central pixel. Consequently, we must consider in computation of the disparity only the pixels belonging to the same region (object). The boundary between two regions is detected using the gradient magnitude information. Thus, the idea is to shape the variable support (window) at each correspondence based on gradient information. Figurel shows a typical case where the window $\mathrm{W}$ contains pixels of two regions (objects).

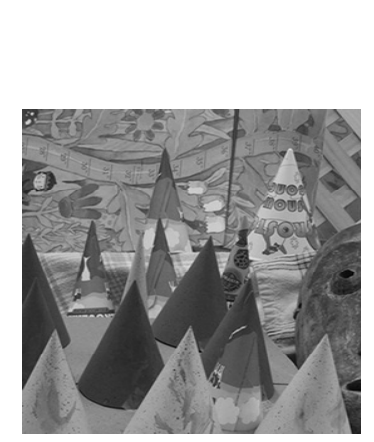

(a)

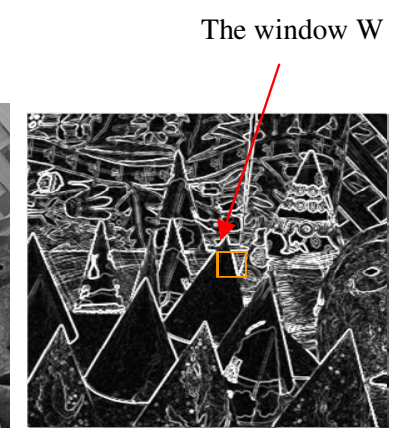

(b)

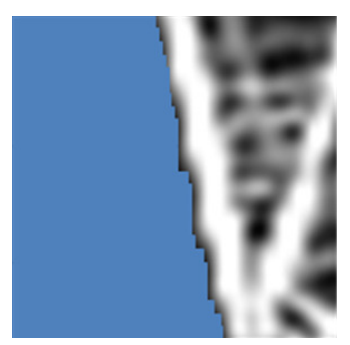

(c)

Figure1. Example of region boundaries problem: (a): The reference image, (b): Case where the window W contains pixels of two regions, (c): The blue color represents the pixels of the same region of the window $\mathrm{W}$ involved in the computation of the disparity.

The use of gradient information allows for including in the aggregation stage also information dealing with the connectiveness of pixels and the shape of the window.

In our work, we assume that the images pairs are rectified. Thus the search for correspondences in the images can be limited to one dimension, ensuring a fast implementation of the stereo matching algorithm. We propose in this section the steps allowing the computation of the initial disparity map using disparity space image (DSI) structure. Finally, in order to keep the good trade-off between accuracy and processing time, a simple method is applied for smoothing the disparity map.

\subsection{Computation of the Disparity Space Image (DSI)}

Disparity Space Image (DSI) is an explicit representation of the matching space introduced by Bobik and Intille [14]. It plays an essential role in the development of the overall matching algorithm which uses the occlusion constraints. Thus, it has the advantage of improving disparities in occluded areas.

Assuming that images pairs are rectified, the search for correspondence of each feature in one image will be done in the same horizontal line of the other image. Thus the disparity computation concerns two matched points which have the same ordinate. For each pixel $\mathrm{p}_{1}\left(\mathrm{x}_{1}\right.$ , $\left.\mathrm{y}_{1}\right)$ in the left image (reference image), the disparity computation will concern all pixels of a window $\mathrm{W}_{1}$ centred on $\mathrm{p}_{1}$. At each pixel $\mathrm{p}_{\mathrm{i}}\left(\mathrm{x}_{\mathrm{i}}, \mathrm{y}_{\mathrm{i}}\right)$ of the $\mathrm{W}_{1}$, the matched pixel $\mathrm{p}_{\mathrm{j}}\left(\mathrm{x}_{\mathrm{j}}, \mathrm{y}_{\mathrm{j}}\right)$ will

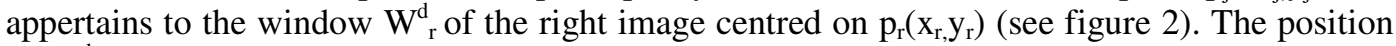
of $\mathrm{W}_{r}^{\mathrm{d}}$ depends on the disparity $\mathrm{d}$ associated to the pair $\left(\mathrm{p}_{1}, \mathrm{p}_{\mathrm{r}}\right)$ which varies from zero to $\mathrm{d}_{\max }$, where $\mathrm{d}_{\max }$ represents the highest disparity value of the stereoscopic images (so-called disparity range).

We have thus: $x_{j}=x_{i}+s * d, y_{j}=y_{i}$, where $\mathrm{s}=\{+1,-1\}$ is a sign chosen so that all disparities are positives. To determine the disparity of a given pixel $\mathrm{p}_{1}\left(\mathrm{x}_{1}, \mathrm{y}_{1}\right)$, we calculate for each disparity $\mathrm{d}$ the score $D S I^{\mathrm{d}}\left(\mathrm{p}_{\mathrm{i}}\right)$ of all pixels $\mathrm{p}_{\mathrm{i}}$ of the adaptive windows $\mathrm{W}_{1}$. 
International Journal of Artificial Intelligence \& Applications (IJAIA), Vol.2, No.3, July 2011

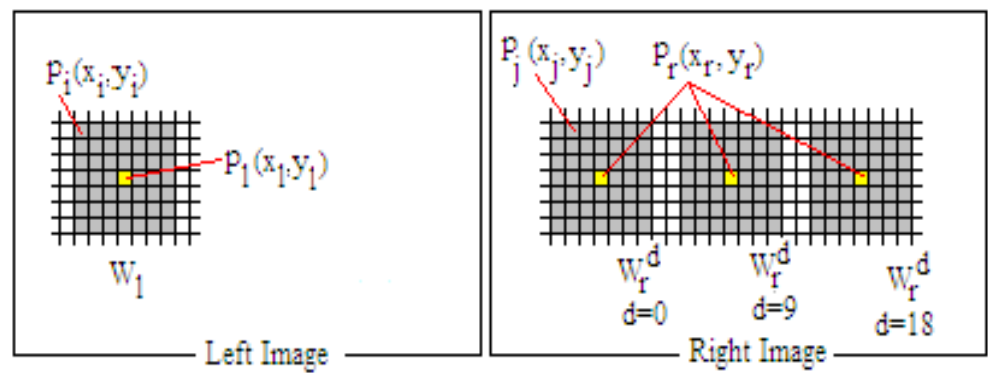

Figure 2. Different windows used for DSI Computation

Twardowski et al. have presented in [28] a survey of the gradient comparison measures reported in the literature given by the following equations:

$$
\begin{aligned}
& E=\left|w_{x l}-w_{x r}\right|+\left|w_{y l}-w_{y r}\right| \\
& E=\left(w_{x l}-w_{x r}\right)^{2}+\left(w_{y l}-w_{y r}\right)^{2}
\end{aligned}
$$

where $w_{a b}$, means $a$ coordinate of gradient vector $b$.

$$
\mathrm{E}=\frac{\left(\left|w_{l}\right|+\left|w_{r}\right|\right)}{2}-\alpha\left|w_{l}-w_{r}\right|
$$

Where $\left|w_{a}\right|$ is the module of vector $a$ and $\alpha$ is the weight parameter.

They also proposed a new matching measure using two coordinates ( $m, \square$ ) of the gradient vectors expressed in the following form:

$$
E=\left|m_{r}-\cos \left(\square_{\mathrm{r}^{-}} \square_{1}\right) m_{l}\right|+\alpha\left|\sin \left(\square_{\mathrm{r}^{-}} \square_{1}\right) m_{l}\right|
$$

Where $m_{r}, m_{l}$ are modules of right and left gradient vectors, $\square_{\mathrm{r}}, \square_{1}$ are angles of right and left gradient vectors and $\alpha$ is a weight parameter. Twardowski et al. have compared these measures [28], the disadvantage of the measure (4) is the computation complexity relative to other measures.

In our case, we added to the matching measure the intensity that gives more robustness. Thus, the matching procedure uses the intensity image, the module and orientation of the gradient.

The $3 \times 3$ Sobel [31] operator is used to compute the gradient values in $x$ and $y$ directions. Denote the intensity of an arbitrary pixel is given by $I(x, y)$, the gradient is defined as:

$$
\left[\begin{array}{l}
G_{x} \\
G_{y}
\end{array}\right]=\left[\begin{array}{l}
\frac{\partial I}{\partial x} \\
\frac{\partial I}{\partial y}
\end{array}\right]
$$

Its magnitude (module) is defined as:

$$
|\mathrm{G}|=\left|\mathrm{G}_{x}\right|+\left|\mathrm{G}_{y}\right|
$$


The orientation $\mathrm{O}(\mathrm{x}, \mathrm{y})$ of the gradient vector is:

$$
\mathrm{O}=\operatorname{tang}^{-1}\left(\mathrm{G}_{x} / \mathrm{G}_{x}\right)
$$

For a given disparity d, the score $D S I^{\mathrm{d}}\left(\mathrm{p}_{\mathrm{i}}\right)$ score is computed as the sum of absolute difference of three attributes (intensity, gradient magnitude and gradient orientation) as follow:

$$
\begin{gathered}
\operatorname{DSI}_{I}^{d}\left(p_{i}\right)=\left|I_{l}\left(x_{i} y_{i}\right)-I_{r}\left(x_{i}+S * d, y_{i}\right)\right| \\
\operatorname{DSI}^{d}{ }_{G}\left(p_{i}\right)=\left|G_{l}\left(x_{i,} y_{i}\right)-G_{r}\left(x_{i}+S * d, y_{i}\right)\right| \\
\operatorname{DSI}^{d} O\left(p_{i}\right)=\left|O_{l}\left(x_{i}, y_{i}\right)-O_{r}\left(x_{i}+S * d, y_{i}\right)\right| \\
\operatorname{DSI}^{d}\left(p_{i}\right)=\operatorname{DSI}_{I}^{d}\left(p_{i}\right)+\operatorname{DSI}_{G}^{d}\left(p_{i}\right)+\operatorname{DSI}_{O}^{d}\left(p_{i}\right)
\end{gathered}
$$

Where $\left(\mathrm{I}_{1}, \mathrm{I}_{\mathrm{r}}\right),\left(\mathrm{G}_{1}, \mathrm{G}_{\mathrm{r}}\right),\left(\mathrm{O}_{1}, \mathrm{O}_{\mathrm{r}}\right)$ are respectively the intensities, gradient magnitudes, gradient orientations values of the pixels on the left and right images.

Aggregation is performed by summing the calculated matching costs over a variable support window with constant disparity $\mathrm{d}$. The aggregation $\operatorname{cost} \mathrm{AC}_{\mathrm{pi}}^{\mathrm{d}}$ is defined as:

$$
A C_{p i}^{d}=\sum_{p i \in S_{p}} D S I^{d}(b i)
$$

Where $S_{p}$ is a set of neighboring pixels on which $p$ lies.

Considering all the aggregation values obtained varying the disparity in the range $\left[0 . . \mathrm{d}_{\max }\right]$, the initial disparity of the pixel $\mathrm{p}_{1}$ is chosen as the disparity $\mathrm{d}^{*}$ with the minimal cost $\mathrm{AC}^{\mathrm{d}^{*}}$ among the various costs of neighboring pixels to $p_{1}$ of the variable support window $W_{1}$ and will be noted $\mathrm{d}^{*}\left(\mathrm{p}_{1}\right)$.

\section{DISPARITY MAP REFINEMENT}

The resulting disparity map described above is not the optimal one because it still some noise and errors. We propose in the following our disparity map refinement method. Even if there exists more accurate techniques for the sub-pixel refinement in the literature [32], [33], and [39] they are computationally too expensive for real-time stereo vision.

\subsection{Refinement Method}

We assume that initial disparities of all pixels of the left image are computed. For each pixel $\mathrm{p}_{1}$ of the left image, we first verify if the disparity is dominant in the variable support window $\mathrm{W}_{1}$. If it is the case, this disparity will be considered as the final disparity and does not necessitate any refinement. Otherwise, we do a refinement which consists to select in variable support window $\mathrm{W}_{1}$ the disparity associated to the best score for each pixel. In this step, we apply a vote in order to choose the dominant disparity in the associated $\mathrm{W}_{1}$ using the central pixel $\mathrm{p}_{1}$ and its 
neighboring pixels. The disparity that will obtain the highest number of confirmations (dominant) will be considered as the new disparity of the central pixel $\mathrm{p}_{1}$.

\subsection{Disparity Map Smoothing}

Finally, in order to keep the good trade-off between accuracy and processing time, a simple median filter is applied for smoothing the disparity map. The median filter is a robust method, often used to remove the impulsive noise known for its salt and pepper noise from an image [34-38]. The median is calculated by first sorting all the pixel values from the surrounding neighbourhood into numerical order and then replacing the pixel being considered with the middle pixel.

\section{EXPERIMENTAL RESULTS}

In this section, we describe the experiments conducted to evaluate the performance of the proposed method. Our aim is to obtain an accurate disparity map and a fast runtime which is the requirement of any obstacle detection system of autonomous mobile robot navigation. To this purpose, an extensive performance evaluation and comparison between different methods is proposed. The two criteria used for the evaluation are then accuracy and computation cost.

For the evaluation of stereo matching algorithms, in our work, we use the Middlebury Stereo Evaluation Ranking [40]. This evaluation uses four stereo image sets. These are the Tsukuba, Venus,Teddy and Cones datasets with the corresponding ground truth.

Figure 3 illustrates the results of the disparity map obtained. From left to right: the first column images are the reference images, the second column images are the Ground truths, the third column images are the initial disparity maps obtained and the four column are the final disparity maps obtained after the refinement step.

Our method was implemented using the $\mathrm{C}++$ language and the timing tests were performed on a Personal computer PC, Core duo 2.2 GHZ.

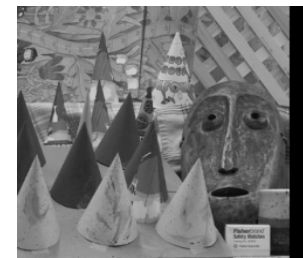

Cones

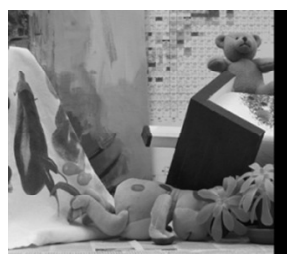

Teddy

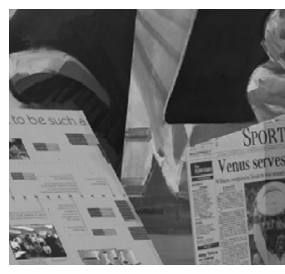

Venus

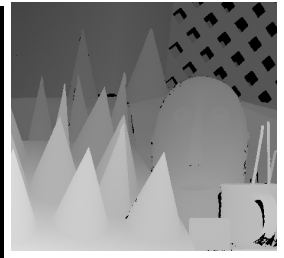

Ground truth disparity

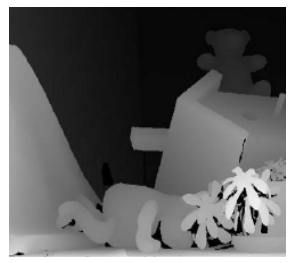

Ground truth disparity

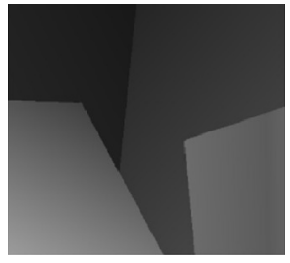

Ground truth disparity

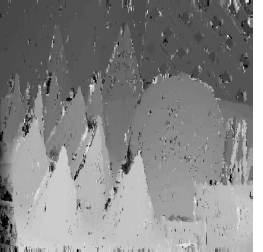

Initial Disparity

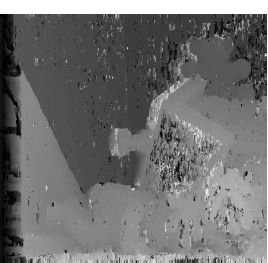

Initial Disparity

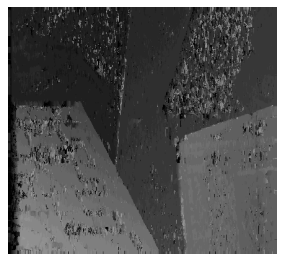

Initial Disparity

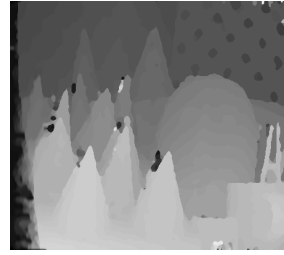

Final Disparity

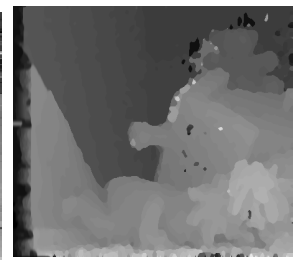

Final Disparity

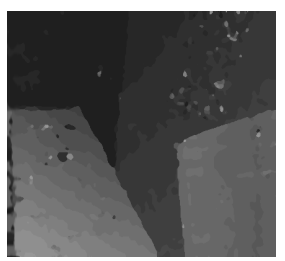

Final Disparity 
International Journal of Artificial Intelligence \& Applications (IJAIA), Vol.2, No.3, July 2011

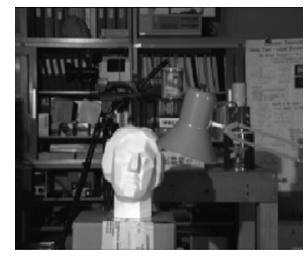

Tsukuba

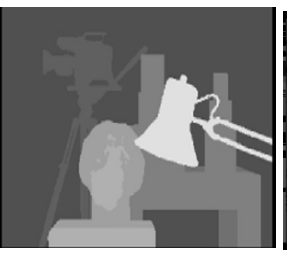

Ground truth disparity

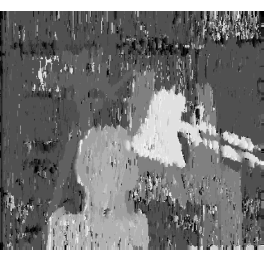

Initial Disparity

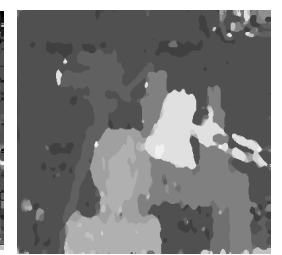

Final Disparity

Figure 3. Disparity map obtained with our method, From left to right: Images reference,

Ground truths, initial disparity maps, and final disparity maps (obtained after the refinement step)

In order to show the efficiency of the use an adaptive support window, we have also evaluated our method using a fixed window $7 \times 7$. Figure 4 and figure 5 show respectively the processing time and the matching quality obtained by the two implementations.

It is clear that, by using a variable support, the processing time has decreased considerably, and the percentage of bad pixels of the disparity map drops approximately by $2,95 \%$ when compared to our method with fixed window.

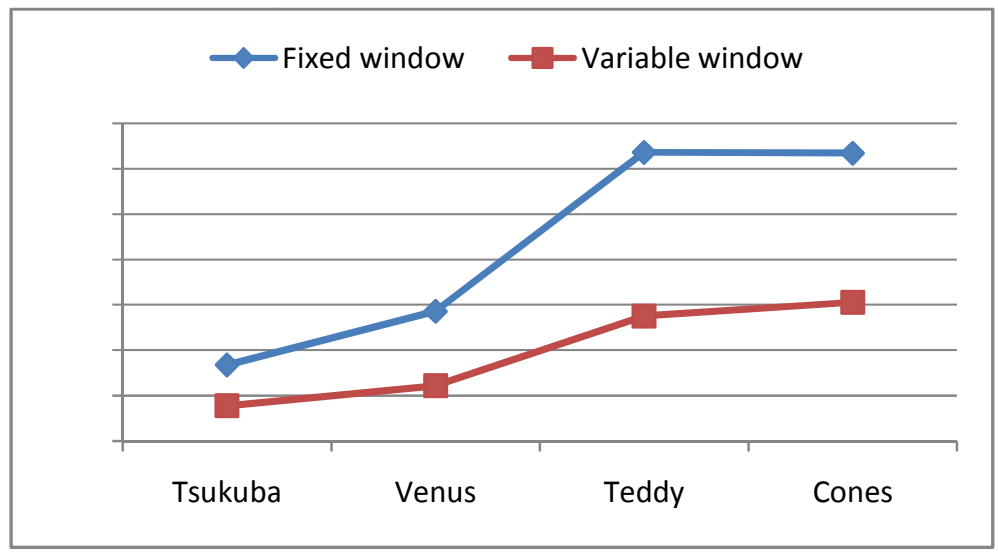

Figure 4. Processing time (second) obtained by the fixed window and variable support window implementations

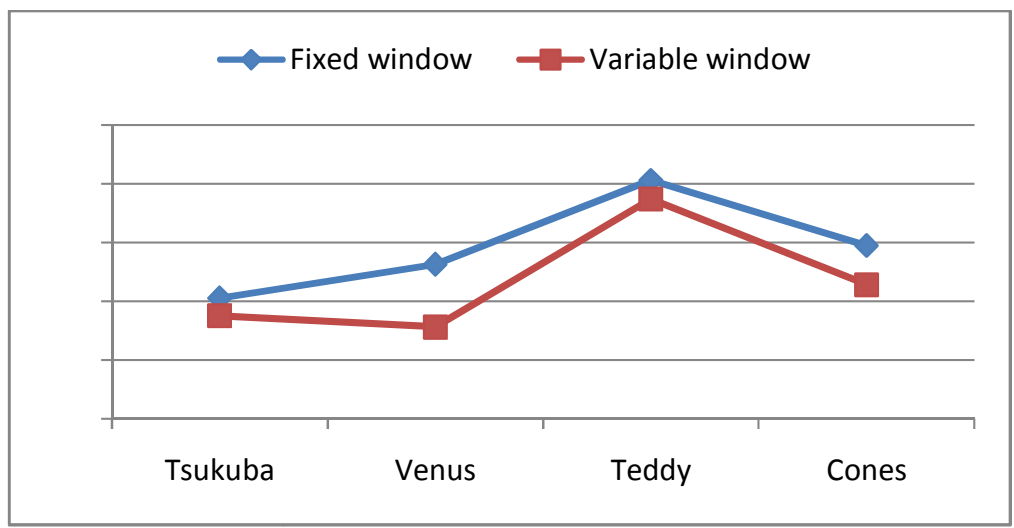

Figure 5. Accuracy (percentage of bad pixels) obtained by the fixed window and variable support window implementations 


\subsection{Discussion and comparison}

This section presents a comparison between the proposed method and other state-of-art methods. Table 1 shows a comparison of stereo vision implementation reported in the literature in terms of computation time. The description of the systems introduced here is restricted to the system platform, the basic matching strategy, the image size and the processing time achieved. All performance data are taken from the authors' papers. A ranking of each method according to the computation time is shown in the second column.

Table 1. Comparison of stereo vision implementations (/ means not available)

\begin{tabular}{|c|c|c|c|c|}
\hline Author & Time(s) & Algorithm & Image size & Machine \\
\hline Tombari [5] & $0.2 \mathrm{~s} \quad 1$ & Aggr. Stra. Based on color segm. & Tsukuba & $2.4 \mathrm{GHz}$ Intel core Duo \\
\hline Gerrits [7] & $2 \mathrm{~s} \quad 3$ & Segment. based & Teddy & 2.4 GHz Intel core Duo \\
\hline Kim [9] & $\begin{array}{ll}4.4 \mathrm{~s} & 4\end{array}$ & Dyn. Prog. & Tsukuba & $2.4 \mathrm{GHz}$ Pentium IV \\
\hline Ogale [4] & $1-5 \mathrm{~s} \quad 5$ & 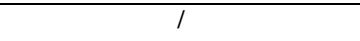 & All images & I \\
\hline Our Method & $\begin{array}{ll}0.2 \mathrm{~s} & \\
0.39 \mathrm{~s} \quad 2\end{array}$ & $\begin{array}{c}\text { DSI + adapt. support } \\
\text { DSI + refinement }\end{array}$ & Tsukuba & $2.2 \mathrm{GHz}$ core Duo \\
\hline Veksler [26] & $6 \mathrm{~s}$ & Graph-cut & Tsukuba & $0.6 \mathrm{GHz}$, Pentium III \\
\hline Tappen [12] & $183 \mathrm{~s} \quad 11$ & Accelerated Belief Prop. & Map & $2.4 \mathrm{GHz}$ Pentium IV \\
\hline Mattocia [6] & $13 \mathrm{~s} \quad 7$ & LC Locally Consist. & Tsukuba & $2.5 \mathrm{GHz}$ Intel Core Duo \\
\hline Yoon [24] & $60 \mathrm{~s}$ & Adapt. Support window-based & Tsukuba & AMD 2700 \\
\hline Vanetti [16] & $100 \mathrm{~s} \quad 9$ & Self org. Map & All images & $1.8 \mathrm{GHz}$ AMD Processor \\
\hline Venkatesh [15] & $120 \mathrm{~s} \quad 10$ & Self org. Map & $256 \times 256$ & $1.4 \mathrm{GHz}$ Pentium IV \\
\hline Gutierrez [25] & Few minutes 12 & Bayesian estimation & $256 \times 255$ & I \\
\hline Tombari [27] & $33 \mathrm{mn} 34 \mathrm{~s} \quad 13$ & Segment Support & Teddy & $2.4 \mathrm{GHz}$ Intel core Duo \\
\hline
\end{tabular}

Scharstein and Szeliski [20] have developed an online evaluation platform, the Middlebury Stereo Evaluation [40], which provides a number of stereo image datasets consisting of the stereo image pair and the appropriate ground truth image.To evaluate an algorithm on this website, disparity maps of all datasets have to be generated and uploaded. The disparity maps have to correspond to the left stereo image and the disparities have to be scaled by a certain factor. The evaluation engine calculates the percentage of bad matched pixels, within a certain error threshold, by pixel-wise comparison with the ground truth image. Many stereo algorithm developers use this platform for evaluation. This gives a significant overview of how the developed algorithm performs in comparison to other algorithms. The platform is up-to-date and constantly growing. Parameters ALL and NOCC are defined according to the Middlebury website [40].

ALL is the error computed on the whole image and NOCC is the error computed on the whole image excluding the occluded region. Among the quality measures proposed by [20] in their paper we adopted the percentage of bad matching pixels between the computed disparity map $d_{C}$ $(\mathrm{x}, \mathrm{y})$ and the ground truth map $\mathrm{d}_{\mathrm{T}}(\mathrm{x}, \mathrm{y})$ :

$$
P B P=\left(1 / N \sum\left(\left|d_{C}(x, y)-d_{T}(x, y)\right|>\delta_{d}\right)\right)
$$

Where $\delta \mathrm{d}$ is the error disparity deviating from the ground truth more than 1 pixel. 
Table 2 shows the comparisons results in terms of accuracy of the disparity maps obtained by some stereo vision methods reported in the literature. We use four reference stereo pairs and for each of them evaluate the error rates on the two ground truth maps Nocc and All. Similarly to the evaluation of computation time, table 2 shows the ranking of methods according to the accuracy. Accuracy corresponds to the percentage of the correct matched pixels. Finally, inspired by the methodology proposed in [22], table 3 reports in the rightmost column the ranking obtained by averaging the overall accuracy ranking and the time ranking, so as to highlight the methods that better trade-off between accuracy and computational efficiency. Overall, by looking at the last column in Table 3 we can observe that our algorithm can be regarded as an interesting trade-off between accuracy and speed.

Table 2. Accuracy according to the methodology defined by the Middlebury website

(/ means not available)

\begin{tabular}{|c|c|c|c|c|c|c|c|c|c|c|}
\hline \multirow[t]{2}{*}{ Method } & \multicolumn{2}{|c|}{ Cones } & \multicolumn{2}{|c|}{ Teddy } & \multicolumn{2}{|c|}{ Tsukuba } & \multicolumn{2}{|c|}{ Venus } & \multirow{2}{*}{\multicolumn{2}{|c|}{$\begin{array}{c}\text { Accuracy } \\
(\%)\end{array}$}} \\
\hline & ALL & NOCC & ALL & NOCC & ALL & NOCC & ALL & NOCC & & \\
\hline Vanetti [16] & 12.4 & 6.31 & 15.73 & 10.41 & 3.76 & 3.38 & 1.42 & 0.98 & 93.21 & \\
\hline Our method & 15.66 & 6.59 & 21.49 & 13.68 & 5.84 & 3.94 & 6.36 & 5.03 & 90.18 & 5 \\
\hline Mattocia [6] & 15.1 & 4.75 & 18.3 & 9.3 & 3.44 & 1.77 & 1.74 & 0.27 & 92.43 & \\
\hline Yoon [24] & 16 & 5.5 & 21.6 & 12.7 & 6.68 & 4.66 & 6.18 & 4.61 & 91.08 & 4 \\
\hline Veksler [26] & 27.3 & 29.6 & 25.5 & 25.9 & 4.86 & 3.12 & 3.87 & 2.42 & 84.68 & 9 \\
\hline Tombari [5] & 1 & 1 & 1 & 1 & 1 & 1 & 1 & 1 & 86.4 & 8 \\
\hline Tombari [27] & 3.77 & 9.87 & 8.43 & 14.2 & 1.25 & 1.62 & 0.25 & 0.64 & 94.9 & 1 \\
\hline Venkatesh [15] & 32.83 & 27.93 & 28.11 & 23.62 & 23.6 & 22.97 & 15.91 & 15.17 & 76.23 & 10 \\
\hline Gallo(17) & 20.2 & 14.1 & 23.9 & 18.3 & 4.97 & 4.55 & 4.55 & 4.28 & 88.14 & 7 \\
\hline Gerrits [7] & 1 & 13.22 & 1 & 15.78 & 1 & 8.18 & 1 & 8.06 & 88.7 & 6 \\
\hline Tappen [12] & 1 & 1 & 1 & 1 & & 4.1 & 1 & I & 1 & \\
\hline Kim [9] & 1 & 1 & 1 & 1 & 1.53 & 1 & 0.94 & 1 & 1 & \\
\hline
\end{tabular}

Table 3. Comparison of stereo vision implementations in terms of accuracy and computation time

\begin{tabular}{|c|c|c|c|}
\hline Method & Rank Time & Rank Accuracy & Average Rank \\
\hline Our method & $\mathbf{2}$ & $\mathbf{5}$ & $\mathbf{3 . 5}$ \\
\hline Mattocia [6] & 5 & 3 & 4 \\
\hline Tombari [5] & 1 & 8 & 4.5 \\
\hline Vanetti [16] & 7 & 2 & 4.5 \\
\hline Gerrits [7] & 3 & 6 & 4.5 \\
\hline Yoon [24] & 6 & 4 & 5 \\
\hline Tombari [27] & 9 & 1 & 5 \\
\hline Veksler [26] & 4 & 9 & 6.5 \\
\hline Venkatesh [15] & 8 & 10 & 9 \\
\hline
\end{tabular}


International Journal of Artificial Intelligence \& Applications (IJAIA), Vol.2, No.3, July 2011

\section{CONCLuSiON}

In this paper, an efficiency aggregation strategy based on DSI structure and gradient information using a variable support has been proposed, that is not just accurate, but also suitable for the deployment in embedded real-time systems. The disparity map computing process is divided into two main steps. The first step deals with computing the initial disparity map, the second step presents a simple and fast method to refine the initial disparity map so an accurate result can be achieved. Using this method (combination of gradient information and DSI structure), we approach the results of global methods without sacrificing the simplicity, flexibility and speed of local aggregation methods. Comparing with a result of a traditional block matching and variable support, our method can be regarded as an interesting trade-off between accuracy and speed. In the future, for parallel calculation, further optimizations of the proposed approach, based on multi-threads, might succeed in order to achieve better performance.

\section{REFERENCES}

[1] Di Stefano, L., Marchionni, M., Mattoccia,S, (2004), "A fast area-based stereo matching algorithm”. Image and vision computing, 22(12), pp. 983-1005.

[2] Maas, R.,Haar Romeny, B., Viergever, M, (1999), “Area-based computation of stereo disparity with model_based window size selection”. Computer Vision and Pattern Recognition (CVPR), pp. 106-112.

[3] Kumar, S., Chatterji, B. (2002) "Stereo matching algorithms based on fuzzy approach. Int. Journal in Pattern Recognit. Artif. Intell. 16,7 , pp. 883-899.

[4] Ogale, A., Aloimonos, Y., (2005) "Shape and the stereo Correspondence Problem". IJCV 65, 3,pp.147-1758.

[5] Tombari, F.,Mattoccia, S., and Di Stefano, L., (2008) "Near real-time based on effective cost aggregation". ICPR.

[6] Mattocia, F.,( 2009) "SA locally global approach to stereo correspondence". ICCV, 12 th Intern. Conf. on Computer Vision Workshops, pp. 1763-1770.

[7] Gerrits , M., Bekaert,P., (2006) "Local stereo matching with segmentation-based outlier rejection. In proceeding Conference on Computer and robot Vision.

[8] Kanade, T.,Okutomi, (1994) M., "A stereo matching algorithm with an adaptive window: theory and experiment”. IEEE Trans. Pattern Anal. Machine Intell., 16, pp. 920-932.

[9] Kim J., Lee K., Coi B. and Lee S., (2005) "A dense stereo matching using two-pass dynamic programming with generalized ground control points”, In Proc. Conf. Computer Vision and Pattern Recognition, pp.1075-1082.

[10] Kolmogorov, V., Zabih, R.,( 2002) "What energy functions can be minimized via graph cuts?". ECCV (3), Lecture notes in computer science, pp.65-81.

[11] Banno , A., Ikeuchi,K. (2009) "Disparity map refinement and 3D Surface smoothing via directed anisotropic diffusion”. 12 th ICCV Workshops,pp. 1870-1877.

[12] Tappen M.,Freeman W., (2003) "Comparison of Graph cuts with Belief Propagation for Stereo, using identical MRF, Parameters”. Inter. Conf. on Computer Vision, ICCV.

[13] Yang Q., Wang L., Yang R., Stewenius H. Nister D.,( 2008) "Stereo Matching with ColorWeighted Correlation, Hierarchical Belief Propagation and Occlusion Handling". PAMI.

[14] Bobik,A., Intille,S.,( 1999) “Large occlusion stereo". International Journal on computer vision 33, ,pp.181-200.

[15] Venkatesh, Y.V,Raja,S.K., Raspanti,M., (2007) "Neural disparity computation for dense twoframe stereo correspondence".IEEE Transaction On Image Processing 16( 11). pp: 2822-2829. 
International Journal of Artificial Intelligence \& Applications (IJAIA), Vol.2, No.3, July 2011

[16] Vanetti, M.,Gallo,I.,Binaghi,E.,(2009). "Dense Two-Frame Stereo Corresponence by SelfOrganizing Neural Network". in Image analysis and processing, LNCS, Springer, pp. 10351042.

[17] Gallo,I., Binaghi, E. and Raspanti, M. (2008) "Neural disparity computation for dense twoframe stereo correspondence". In Int. journal Pattern Recognition Letters 29, pp. 673-687.

[18] Zickler, T. E. Ho, J. ,Kriegman, D. J. Ponce ,J. Belhumeur, P. N. (2003) "Binocular Helmholtz Stereopsis". In IEEE Conference Proceedings of International Conference on Computer Vision, ICCV,volume 2, pp. 1411-417.

[19] Lhuillier, M. , Quan, L. (2000) "Robust Dense Matching Using Local and Global Geometric Constraints". In IEEE Conference Proceedings of International Conference on Pattern Recognition,ICPR, , volume 1, pp. 968-972.

[20] Scharstein, D., Szeliski,R., (2002) "A taxonomy and evaluation of dense two-frame stereo correspondence algorithms". Int. Journal on Computer Vision, pp. 47,7-42.

[21] Nalpantidis, L., Sirakoulis, G.,and Gasteratos, (2008) A. "Review of stereo matching algorithms: from software to hardware”. International journal of optomechtonics 2:,pp. 435-462 .

[22] Tombari, F.,Mattoccia, S.,and Di Stefano, L., (2008) “Clasification and evaluation of cost aggregation methods for stereo correspondence”. In Proc. CVPR.

[23] Binaghi,, E., Gallo,I., (2004) Fornasier, C., Raspanti,M. "Neural adaptative stereo matching”. Pattern Recognition Letters 25,..pp. 1743-1758.

[24] Yoon,K., Kweon,I., (2006) “Adaptive Support Weight Approach for Correspondence Search”. IEEE Tran. On Pattern Analysis and Machine Intelligence, vol. 28.

[25] Gutierrez, S., Marroquin,J.,( 2004) "Robust approach for disparity estimation in stereo vision. Image and Vision Computing, pp.83-195.

[26] Veksler, O., (2003) "Extracting dense features for visual correspondence with graph cuts". Proc. Of the IEEE Computer Society Conference on Computer Vision and pattern Recognition.

[27] Tombari, F.,Mattoccia, S., and Di Stefano, L., (2007) "Segmentation-based adaptive support for accurate stereo correspondence". In Proc. Pacific-Rin Symposium on Image and Video Technology.

[28] Twardowski, T., Cyganek, B., Borgosz, J. (2004) "Gradient based dense stereo matching", Lecture Notes in Computer Science 3211 (8) pp.721-728.

[29] Hirscmuller, H. Innocent P. and J.Garibaldi. (2002), "Real-time correlation-based stereo vision with reduced border errors". IJCV, 47:1-13.

[30] Gong, M. Yang, R..Liang W and Gong, M. (2007), “A performance study on different cost aggregation approaches used in real-time stereo matching ”, IJCV,75 (2), :283-296.

[31] Gonzalez R.C., Woods, R.E. ( 2002), Digital Image Processing, second ed. Pearson Education International.

[32] Shimizu, M., Okutomi,M.,( 2003) Precise sub-pixel estimation on area based matching", in Proceedings of the eight IEEE International Conference on Computer Vision.

[33] Psarakis, E. Z., Evangelidis, G. D. (1997) "A generic implementation framework for FPGA based stereo matching" in Proceedings of the IEEE Region 10th Annual Conference on Speech and Image Technologies for Computing and Telecommunications.

[34] Arce, G.R., (2005) Nonlinear Signal Processing: A Statistical Approach, Wiley:New Jersey, USA.

[35] Egnal, G., Wildes, R. P. (2002) "Detecting Binocular Half-Occlusions : Empirical Comparisonsof Five Approaches". IEEE Transactions on Pattern Analysis and Machine Intelligence, PAMI,24(8), , pp.1127-1133. 
International Journal of Artificial Intelligence \& Applications (IJAIA), Vol.2, No.3, July 2011

[36] Koschan, A. (1993) "Dense Stereo Correspondence Using Polychromatic Block Matching". In proceedings of International Conference on Computer Analysis of Images and Patterns, CAIP, volume 719 of Lecture Notes in Computer Science, pp. 538-542.

[37] Muhlmann, K., Maier, D., Hesser, J. Manner. R. M, (2001) "Calculating Dense Disparity Maps from Color Stereo Images, an Efficient Implementation". In IEEE Conference Proceedings of Workshop on Stereo and Multi-Baseline Vision, SMBV,pp. 30-36,

[38] Eklund, M. P.et A. A. Farag. (2003) "Robust correspondence methods for stereo vision". International Journal of Pattern Recognition and Artificial Intelligence, PRAI, 17(7) , , pp.10591079.

[39] Reji A. P and Tessamma T. (2010) "Single frame image super resolution using learned directionlets" International Journal of Artificial Intelligence \& Applications (IJAIA), Vol.1, No.4, pp. 29-42.

[40] http://vision.middlebury.edu/stereo. Middlebury stereo evaluation.

\section{Authors}

Nadia BAHA received the Magister in Computer Science at CDTA in 1991. She is currently a $\mathrm{PhD}$ student and a researcher at the Computer Science Institute of University of Sciences and Technologies, USTHB, Algiers, Algeria. She is an author of numerous publications for conferences, proceedings. Her research interests include computer vision and mobile robot navigation.

Slimane LARABI received the Ph.D. in Computer Science from the Polytechnic National Institute of Toulouse, France, in 1991. He is currently Professor at the Computer Science Institute of University of Sciences and Technologies. Algiers, Algeria. He is the head of Computer Vision group at the laboratory of artificial Intelligence of the same university, and an author of numerous publications for conferences, proceedings, and journals. 